\section{Amostras complexas em inquéritos populacionais: planejamento e implicações na análise estatística dos dados}

\section{Complex Sampling Design in Population Surveys: Planning and effects on statistical data analysis}

\section{Célia Landmann Szwarcwald Giseli Nogueira Damacena}

Laboratório de Informação em Saúde, Instituto de Comunicação e Informação Científica e Tecnológica em Saúde, Fundação Oswaldo Cruz.

Correspondência: Célia Landmann Szwarcwald, Laboratório de Informação em Saúde, Instituto de Comunicação e Informação Científica e Tecnológica em Saúde, Fundação Oswaldo Cruz, Av. Brasil, 4365 - Pavilhão Haity Moussatché - Manguinhos, Rio de Janeiro, RJ, CEP 21040-900. E-mail: celials@cict.fiocruz.br

\section{Resumo}

A avaliação do desempenho dos sistemas de saúde das nações vem ganhando importância crescente entre os gestores do setor saúde. Entre os instrumentais de avaliação do desempenho de saúde, destacam-se os inquéritos nacionais de saúde, cada vez mais utilizados para avaliar o estado de saúde da população e a assistência de saúde prestada do ponto de vista do usuário. A maioria dos inquéritos nacionais de saúde não usa amostragem aleatória simples, em parte por restrições orçamentárias, em parte por limites de tempo associado à coleta de dados. Em geral, utiliza-se combinação de vários métodos probabilísticos de amostragem para seleção de uma amostra representativa da população, chamada de desenho complexo de amostragem. Entre os métodos de amostragem mais utilizados conjuntamente, destacam-se a amostra aleatória simples, a amostragem estratificada, e a amostragem por conglomerados. Resultante desse processo, a preocupação subseqüente é a análise de dados provenientes de amostras complexas. Este artigo trata de questões relacionadas à análise estatística de dados obtidos através de pesquisas com desenhos complexos de amostragem. Apresentam-se os problemas que ocorrem quando a análise estatística não incorpora a estrutura do plano amostral. Ao ignorar o desenho de amostragem, a análise estatística tradicional, sob a suposição de amostragem aleatória simples, pode produzir incorreções tanto para as estimativas médias como para as respectivas variâncias, comprometendo os resultados, os testes de hipóteses e as conclusões da pesquisa. Para a exemplificação dos métodos, é utilizada a Pesquisa Mundial de Saúde (PMS), realizada no Brasil em 2003.

Palavras-chave: Inquérito. Amostragem. Desenho complexo. Análise estatística. Brasil. 


\section{Abstract}

The evaluation of the health systems of nations has been gaining increasing importance among health managers. Among the evaluation tools for the performance of health systems, nationwide health surveys have been more and more used to evaluate the health status of the population and satisfaction with healthcare from the user's point of view. Most national health surveys do not use simple random sampling, either due to budget restrictions or because time constraints associated with data collection. In general, a combination of several probabilistic sampling methods is used to select a representative sample of the population, which is called complex sampling design. Among the several sampling techniques, the most frequently used are simple random sampling, stratified sampling and cluster sampling. As a result of this process, the next concern is the statistical analysis of the data from complex samples. This paper deals with issues related to data analysis obtained from surveys using complex sampling designs. It discusses the problems that arise when the statistical analysis does not incorporate the sampling design. When the design is neglected, traditional statistical analysis, based on the assumption of simple random sampling, might produce improper results not only for the mean estimates but also for standard errors, thus compromising results, hypothesis testing, and survey conclusions. The World Health Survey (WHS) carried out in Brazil, in 2003, is used to exemplify complex sampling methods.

Keywords: Survey. Sampling. Complex design. Statistical analysis. Brazil.

\section{Introdução}

A avaliação do desempenho dos sistemas de saúde das nações vem ganhando importância crescente entre os gestores do setor saúde, sendo considerada como etapa essencial para o planejamento das ações voltadas para garantir a qualidade da atenção e subsidiar decisões que atendam às necessidades da população ${ }^{1}$.

Entre os instrumentais de avaliação do desempenho de saúde, destacam-se os inquéritos nacionais de saúde, cada vez mais utilizados para avaliar a assistência de saúde prestada do ponto de vista do usuário. Este tipo de pesquisa caracterizase por entrevistas domiciliares, que abordam dimensões relativas à situação de saúde, como a percepção do estado de saúde e a prevalência de problemas selecionados, questões sobre as atitudes e práticas de risco, além dos aspectos relacionados ao uso dos serviços de saúde, como o acesso e a utilização de serviços, a cobertura de ações de saúde, e a satisfação dos usuários com o funcionamento da assistência de saúde².

Captando informações que, na maioria das vezes, não estão disponíveis nos sistemas nacionais de informações, e permitindo explorar as inter-relações entre as diversas dimensões da saúde junto às características demográficas e socioeconômicas, os inquéritos são de grande importância para o monitoramento da situação de saúde da população, para subsidiar o planejamento das ações e investimentos, bem como para a avaliação das políticas de saúde ${ }^{3}$.

A inferência estatística, em sua abordagem clássica, fundamenta-se na amostra aleatória simples, método que requer que cada membro da população tenha uma chance igual e independente de ser selecionado ${ }^{4}$. Entretanto, a maioria dos inquéritos nacionais de saúde não usa amostragem aleatória simples, em parte por restrições orçamentárias, em parte por limites de tempo associados à coleta de uma grande quantidade de informações ao 
longo de um território geográfico grande. Em decorrência disso, outros métodos probabilísticos são geralmente utilizados nos inquéritos de base populacional, como a amostragem estratificada e a amostragem por conglomerados em múltiplos estágios com probabilidades desiguais de seleção ${ }^{5}$, para prover uma amostra representativa da população em tempo hábil e de acordo com o orçamento previsto ${ }^{6}$.

A combinação de vários métodos probabilísticos de amostragem para seleção de uma amostra representativa da população é chamada de desenho complexo de amostragem. Resultante deste processo, a preocupação subseqüente é a análise de dados provenientes de amostras complexas. Este tipo de análise tem sido objeto de estudo e discussão nas literaturas nacional e internacional recentes ${ }^{7-9}$. Algumas publicações mostram os problemas que ocorrem quando a análise estatística não incorpora a estrutura do plano amos$\operatorname{tral}^{10}$. Ao ignorar o desenho de amostragem, a análise estatística tradicional, sob a suposição de amostragem aleatória simples, pode produzir incorreções, tanto para as estimativas médias como para as respectivas variâncias, comprometendo os resultados, os testes de hipóteses e as conclusões da pesquisa ${ }^{11}$.

Este artigo trata de questões relacionadas à análise estatística de dados obtidos através de pesquisas com desenhos complexos de amostragem. Para a exemplificação dos métodos, é utilizada a Pesquisa Mundial de Saúde, realizada no Brasil em $2003^{12}$.

\section{Elementos dos desenhos complexos de amostragem}

$\mathrm{Na}$ amostragem probabilística, cada indivíduo da população tem uma probabilidade conhecida e não nula de ser selecionado. São vários os métodos de amostragem probabilística, sendo os mais utilizados a amostra aleatória simples, a amostragem estratificada, e a amostragem por conglomerados ${ }^{5}$.
A amostragem estratificada consiste em dividir a população em subgrupos homogêneos para determinadas características e selecionar uma amostra em cada um deles, separadamente. Estes subgrupos não se interceptam e totalizam a população. Cada uma das subdivisões populacionais é denominada de estrato. Este tipo de amostragem é recomendado quando se deseja obter estimativas com certa precisão para cada uma das subdivisões.

Cochran $^{5}$ demonstra que o uso de estratos homogêneos pode resultar em um ganho na precisão de estimativas de características da totalidade da população. Intuitivamente, se cada estrato for homogêneo, a estimativa de uma determinada característica pode ser obtida dentro do estrato com um número relativamente pequeno de observações. Estas estimativas são então ponderadas, adequadamente, para obtenção da estimativa populacional.

$\mathrm{Na}$ amostragem por conglomerados, a unidade amostral é um conjunto de elementos da população. Pelas dificuldades de custo e de obtenção de listas dos elementos da população, a aplicação da amostragem por conglomerados é muito freqüente nos inquéritos de base populacional. Geralmente, este tipo de amostragem é repetido em múltiplos estágios, quando uma amostra de conglomerados é selecionada em cada etapa. Os conglomerados são unidades compostas de subunidades, que vão sendo selecionadas em cada estágio até chegar ao objeto de interesse da pesquisa ${ }^{5}$. Por exemplo, no Brasil pode-se selecionar no primeiro estágio os setores censitários, denominados de unidades primárias de seleção. No segundo estágio, dentro dos setores selecionados, os domicílios, e no último o indivíduo a ser pesquisado.

Particularmente, um método freqüentemente utilizado para a seleção dos conglomerados é a amostragem com probabilidade proporcional ao tamanho (PPT). Neste caso, as unidades primárias são selecionadas com probabilidades proporcionais ao seu tamanho. Este método, além da facilidade de aplicação, tem a vantagem 
de contribuir para a redução da variância entre as unidades de seleção ${ }^{5}$.

Além dos tipos de amostragem e da combinação entre eles, constituindo desenhos complexos de amostragem, outro aspecto merecedor de atenção são as probabilidades desiguais de seleção das unidades em cada um dos estágios. Para compensar as probabilidades desiguais de seleção, são atribuídas ponderações diferenciadas aos elementos da amostra, chamadas de fatores naturais de expansão, correspondentes ao inverso do produto das probabilidades de inclusão nos diversos estágios de seleção.

Finalmente, para ajustar os pesos naturais do desenho e/ou corrigir os problemas originados pela ausência ou recusa de resposta é necessário fazer a calibração para totais conhecidos da população.

A calibração dos fatores naturais de expansão consiste em estimar novos pesos para cada elemento da amostra, através de ajuste dos pesos naturais do desenho segundo informações de variáveis auxiliares da amostra $^{13}$. Por exemplo, um dos propósitos da calibração é obter distribuições amostrais das variáveis auxiliares semelhantes às distribuições populacionais obtidas pelo censo.

\section{Questões relacionadas à análise estatística de dados}

Na utilização de informações coletadas em inquéritos com amostras complexas, uma das preocupações refere-se à análise apropriada dos dados, que não podem ser tratados como se fossem observações independentes e identicamente distribuídas (amostragem aleatória simples).

$\mathrm{Na}$ análise, os seguintes aspectos devem ser considerados ${ }^{11}$ :

- probabilidades distintas de seleção das unidades;

- $\quad$ efeito de conglomeração das unidades;

- estratificação;

- calibração para ajuste de não resposta e outros ajustes para totais conhecidos da população.
O plano de amostragem, processo usado para selecionar a amostra da população, é importante para a estimação dos parâmetros, já que a estimação baseada no plano amostral é fundamentada na ponderação de cada elemento da amostra pelo inverso da sua probabilidade de seleção ${ }^{11}$.

Quando os dados são utilizados para fins descritivos, as estimativas pontuais dos parâmetros populacionais (como totais, médias, proporções) são influenciadas apenas pelos pesos amostrais determinados pelo plano de amostragem. Na maioria das vezes, porém, são usados com fins analíticos, requerendo a estimação de medidas de variabilidade (como a variância e o desviopadrão) para o cálculo dos intervalos de confiança e a realização de testes de hipóteses ${ }^{8}$.

Nos estudos analíticos, as estimativas pontuais são associadas à precisão, dada pelo tamanho do intervalo de confiança. Quanto maior o intervalo, ou quanto maior o erro padrão, menor a precisão da estimativa. A estimação do erro padrão é, pois, essencial para a inferência estatística.

Em planos de amostragem complexa, a estimação da variância é influenciada não somente pelos pesos, mas também pela estratificação e conglomeração, conjuntamente. No que se refere à estratificação, se esta for adequadamente utilizada, a variância da média estimada é, geralmente, inferior à variância se a amostragem fosse aleatória simples, ou seja, são obtidos ganhos de precisão nas estimativas médias ${ }^{5}$.

Já o efeito da conglomeração é, em geral, inverso, produzindo variâncias maiores do que as calculadas com observações independentes. Na amostragem por conglomerados, a variância da média é função direta da variância total e do coeficiente de correlação intra-classe, que mede a correlação entre as observações dentro dos conglomerados. Significa dizer que quanto maior a homogeneidade dentro dos conglomerados, maior a variabilidade entre conglomerados, e maior a variância da média. Portanto, a amostragem por conglomerados acarreta, em geral, a perda de precisão das estimativas médias. 
Para medir o efeito do plano amostral (EPA) sobre a variância das estimativas médias, utiliza-se o chamado "efeito de desenho”, calculado pela razão entre a estimativa da variância determinada pelo plano amostral e a estimativa da variância obtida por uma amostra aleatória simples de mesmo tamanho ${ }^{14}$. Além de ser utilizado para verificar a perda de precisão da estimativa, o efeito de desenho é utilizado também para o planejamento de estudos futuros, no cálculo do tamanho de amostra.

\section{Desenho da Pesquisa Mundial de Saúde no Brasil, 2003}

Como parte de um projeto desenvolvido pela Organização Mundial da Saúde (OMS), dirigido à avaliação de desempenho dos sistemas de saúde dos países membros, a Pesquisa Mundial de Saúde foi realizada no Brasil sob a forma de um inquérito populacional em 5.000 domicílios escolhidos por amostragem probabilística, em âmbito nacional, no período de janeiro a setembro de 2003 .

Através de um questionário modular, a pesquisa considerou diversos aspectos da saúde, tais como: a descrição do estado de saúde; fatores de risco (fumo, álcool, atividade física, nutrição, fatores ambientais); situações crônicas e agudas de saúde; uso de medicamentos; cobertura de programas de saúde (saúde bucal, assistência pré-natal e saúde materno-infantil); avaliação da resposta do sistema do ponto de vista do usuário; gastos relativos das famílias em saúde, incluindo planos privados de saúde.

Com o apoio da Fundação Instituto Brasileiro de Geografia e Estatística (IBGE) foram obtidos os elementos necessários à seleção de uma amostra de setores, bem como os mapas e folhas de coleta do Censo Demográfico $2000^{15}$ dos setores selecionados.

A amostra foi selecionada em três estágios (setores, domicílios e adulto), totalizando 5.000 domicílios e 5.000 indivíduos com 18 anos ou mais de idade. No primeiro estágio foram selecionados 250 setores censitários com probabilidade proporcional do seu número de domicílios particulares permanentes (DPP), sendo excluídos da população amostrada os setores censitários localizados nas áreas rurais da macro-região Norte e os especiais (quartéis, bases militares, alojamentos, acampamentos, embarcações, penitenciárias, asilos, orfanatos, conventos ou hospitais). No segundo, foram selecionados 20 DPPs com eqüiprobabilidade e dentro de um esquema de amostragem inversa. E, no último estágio, foi selecionado um adulto (18 anos ou mais de idade) em cada domicílio para responder à entrevista individual ${ }^{16}$.

As unidades primárias de seleção (setores censitários) foram estratificadas segundo o tamanho da população do município e sua situação (urbana ou rural), gerando seis estratos. O tamanho da amostra de setores foi alocado entre os estratos de forma proporcional à população de cada estrato em 2000, assegurando o número mínimo de cinco setores por estrato.

Em cada estrato, a seleção dos setores censitários foi realizada de forma sistemática e com probabilidade proporcional ao número de domicílios particulares permanentes (DPP) em cada setor. Com o intuito de garantir a representatividade de todos os níveis socioeconômicos do estrato, foi utilizada para estratificação implícita a renda média dos responsáveis pelos domicílios do setor ${ }^{17}$, isto é, previamente à seleção sistemática, os setores foram ordenados em ordem crescente de renda. Os 250 setores selecionados distribuíram-se em 188 municípios, com o número de setores por município variando de um a 14 .

No segundo estágio, foram selecionados, com eqüiprobabilidade, 60 endereços de DPP existentes na folha de Coleta do Censo Demográfico 2000 de cada setor da amostra, para obter 20 entrevistas realizadas em cada setor, seguindo um procedimento seqüencial de visitas, conhecido como amostragem inversa. Este tipo de amostragem, originalmente proposto por 
Haldane $^{18}$, consiste em verificar quantas unidades precisam ser observadas para que seja obtido o número prefixado de sucessos, ou seja, o número prefixado de entrevistas realizadas. Na PMS, sua aplicação consistiu em visitar seqüencialmente os domicílios previamente selecionados, registrar as ocorrências (entrevista ou nãoentrevistas), até que fosse atingido o número previsto de 20 entrevistas realizadas para o setor.

No terceiro estágio, em cada domicílio, um adulto (18 anos ou mais de idade) foi selecionado com probabilidade igual a todos os moradores adultos do domicílio. Prevendo-se até 12 moradores adultos, foram definidos 11 conjuntos de números naturais, começando por $\{1,2\}$ e terminando em $\{1,2, \ldots, 12\}$; e, em cada conjunto, foram feitas 5.000 seleções eqüiprováveis de apenas um dos elementos do conjunto. Os resultados dessas seleções foram colocados na forma de tabelas, que foram incluídas na Folha de Informações sobre Setores, Domicílios e Pessoas a Entrevis$\operatorname{tar}$ (FIS) fornecida ao entrevistador ${ }^{15}$.

Os fatores naturais de expansão foram baseados no plano de amostragem, determinados a partir das probabilidades de inclusão de setor, domicílio e adulto. O cálculo das probabilidades de seleção do setor e do adulto foi o usado, habitualmente, para desenhos com seleção de unidades amostrais com probabilidade proporcional ao tamanho e eqüiprobabilidade, respectivamente. Já a probabilidade de seleção do domicílio teve que ser formulada especificamente, pois foi utilizado esquema de amostragem inversa. Como o uso da folha de Coleta do Censo Demográfico 2000 implicou a seleção de domicílios inexistentes no momento da pesquisa e de alguns domicílios fechados, a probabilidade de inclusão de um domicílio, condicionada à seleção do setor, teve que ser subdividida em três probabilidades:

- a de ser visitado;

- a de pertencer à população amostrada ou de ser elegível; e

- a de ser um dos 20 primeiros domicíli- os elegíveis a concordar com a realização da entrevista ${ }^{16}$.

Finalmente, os fatores de expansão foram calibrados para assegurar coerência com os totais populacionais por grupos de macro-regiões, quintos de renda, sexo e grupos de idade, por meio de estimadores de regressão ${ }^{16}$.

A título de ilustração, na Tabela 1 são apresentadas estimativas pontuais sem e com uso de ponderação, bem como as estimativas do erro padrão e dos efeitos de desenho para algumas variáveis de interesse do estudo. Tais estimativas foram calculadas utilizando o pacote SUDAAN ${ }^{19}$, incorporando os efeitos do plano de amostragem e dos ajustes da calibração dos pesos naturais.

Na Tabela 1, verifica-se que as estimativas pontuais dos parâmetros populacionais (proporções), para todas as variáveis apresentadas, são influenciadas apenas pelos pesos amostrais determinados pelo plano de amostragem, e se mantiveram iguais quando estimadas com ponderação e levando em consideração o efeito de desenho. Já os valores maiores do erro padrão para todas as variáveis indicam que ignorar o plano amostral na estimação da variância leva a estimativas viciadas e incorretas.

Os resultados mostram, pois, que o estimador "ingênuo" da variância, obtido ignorando o plano amostral complexo, leva a subestimar a variância verdadeira do estimador de proporção de diversas variáveis consideradas na PMS brasileira.

\section{Comentários finais}

Embora o tamanho da PMS brasileira tenha sido pequeno, limitado pelos recursos disponíveis (providos pela OMS), de maneira geral pode-se dizer que os resultados obtidos foram fidedignos e comparáveis aos obtidos em inquéritos de maior porte. A exemplificação é dada pela proporção de indivíduos que têm plano privado de saúde, estimada com o mesmo valor que o obtido na Pesquisa Nacional por Amostra de Domicílios, 2003 ${ }^{20,21}$. 
Tabela 1 - Estimativas pontuais e erro-padrão sem e com uso de ponderação e com efeito de desenho para algumas variáveis da Pesquisa Mundial de Saúde. Brasil, 2003

Table 1 - Specific estimates and standard error with and without weighing and with design effect for some variables of the World Health Survey. Brazil. 2003.

\begin{tabular}{|c|c|c|c|c|c|c|}
\hline \multirow[t]{2}{*}{ Variáveis de estudo } & \multicolumn{2}{|c|}{ Sem ponderação } & \multicolumn{2}{|c|}{ Com ponderação } & \multicolumn{2}{|c|}{$\begin{array}{l}\text { Com ponderação e } \\
\text { efeito do plano amostral }\end{array}$} \\
\hline & $\%$ & Erro Padrão & $\%$ & Erro Padrão & $\%$ & Erro Padrão \\
\hline Auto-avaliação da saúde muito boa ou boa & 53,0 & 0,706 & 53,3 & 0,705 & 53,3 & 0,953 \\
\hline Diagnóstico de depressão & 20,1 & 0,568 & 19,3 & 0,559 & 19,3 & 0,769 \\
\hline Obesidade & 10,5 & 0,459 & 10,1 & 0,450 & 10,1 & 0,541 \\
\hline Fumo diário & 22,9 & 0,594 & 22,4 & 0,589 & 22,4 & 0,781 \\
\hline Uso de serviço de saúde no último ano & 68,5 & 0,656 & 68,6 & 0,656 & 68,6 & 0,941 \\
\hline Teve problema bucal e recebeu tratamento & 24,1 & 0,605 & 24,7 & 0,610 & 24,7 & 0,830 \\
\hline
\end{tabular}

Fonte: Pesquisa Mundial de Saúde (PMS). Brasil; 2003

Source: World Health Survey (WHS). Brazil. 2003.

Entretanto, foram necessários ajustes para as estimativas regionais. Como a amostra não foi selecionada para fornecer dados para as macro-regiões do Brasil, a estratificação implícita por renda média dos responsáveis pelos domicílios do setor provocou uma concentração dos setores mais ricos nas regiões Sul e Sudeste e dos mais pobres nas demais regiões. O uso apenas dos pesos naturais do plano de amostragem enviesaria, portanto, os resultados regionais por não representarem o espectro de variação da renda em cada região. Diante desta situação, a calibração dos pesos naturais foi essencial para corrigir esta situação e gerar fatores de expansão que permitissem desagregar os dados nacionais $^{16}$.

Em relação às estimativas para a população total por faixa etária, os fatores naturais de expansão conduziram a uma distribuição um pouco diferente daquela obtida no Censo Demográfico de 2000. Observou-se uma tendência de subestimação das classes de idade mais jovens (até 39 anos) e a superestimação das faixas etárias mais velhas, principalmente de idosos. Em relação à distribuição por sexo, ocorreu superestimação da população adulta feminina. Já a distribuição por quintos de renda mostrou padrão semelhante ao descrito para a população total.

Estes resultados refletem tendências já amplamente conhecidas nas pesquisas de base domiciliar. Ainda que a seleção do morador a ser entrevistado tenha sido realizada por meio de uma tabela de números eqüiprováveis, especialmente preparada para a PMS brasileira e que o treinamento tenha sido rigoroso no sentido de seguir o protocolo de coleta, a presença mais freqüente de mulheres e indivíduos idosos no domicílio acarretou um viés na distribuição da população adulta por faixa etária e sexo. Tendo em vista que o objetivo principal da pesquisa era estimar variáveis relacionadas à saúde na população brasileira, a calibração dos pesos naturais foi necessária para lidar com os problemas de coleta e seleção.

Uma inovação realizada no plano de amostragem da PMS foi a utilização de um esquema de amostragem inversa no último estágio de seleção. Segundo Vasconcellos et al. ${ }^{16}$, este método é chamado de amostragem inversa porque, em vez de definir o número de domicílios a visitar para tentar a entrevista, define-se o número de entrevistas a serem realizadas, trazendo a vantagem de tornar desnecessária a correção do tamanho de amostra calculado para compensar a taxa esperada de não-resposta.

Em todos os 250 setores da amostra, foram obtidas as 20 entrevistas previstas. Todavia, foi necessário visitar, em média, 34,4 domicílios, incluindo 20 domicílios entrevistados $(58,1 \%), 8,5$ recusas de domicílio ou do adulto selecionado (24,7\%), 3,3 domicílios vagos ou que não tinham 
adultos (9,6\%), e 2,6 domicílios inexistentes ou não eram mais domicílios particulares $(7,6 \%)$. Observou-se, ainda, que quanto maior a renda média do setor, tanto maior a proporção de recusas.

O número médio de domicílios visitados por setor indica que o tamanho da amostra de um desenho tradicional deveria ser inflacionado em $70 \%$ para compensar as perdas e obter, em média, 20 entrevistas por setor. No entanto, este tamanho de amostra seria insuficiente para cerca de $30 \%$ dos setores, onde foram necessárias 40 ou mais visitas, porém demasiadamente grande para os demais setores, onde foram visitados menos de 30 domicílios. Nesse sentido, o esquema de amostragem inversa funcionou como uma boa alternativa não somente para baratear os custos, mas também para a realização do mesmo número de entrevistas em todos os setores.

Em relação à incorporação do plano de amostragem na análise estatística dos dados, os resultados aqui apresentados indicam que as estimativas pontuais sofreram poucas alterações com a inclusão das ponderações amostrais. Porém, os efeitos de conglomeração produziram impacto significativo na precisão das estimativas, e poderiam levar a conclusões incorretas na análise de testes de hipóteses.

\section{Referências}

1. Birch S, Gafni A. Achievements and challenges of medicare in Canada: Are we there yet? Are we on course? Int J Health Serv 2005; 35(3):443-63.

2. Chester LGC, Figueiredo GM, Westphal MF, Cardoso MRA, Costa MZA,Gattás VL. Morbidade Referida e Utilização de Serviços de Saúde em Localidades Urbanas Brasileiras: Metodologia. Rev Saúde Pública 1996; 30:153160.

3. De Bruin A, Picavet HS, Nossikov A. Health interview surveys. Towards international harmonization of methods and instruments. WHO Reg Publ Eur Ser 1996; 58:i-xiii, 1-161.

4. Zar JH. Biostatistical Analysis, $3^{\text {rd }}$ Edition. New Jersey: Prentice Hall; 1996.

5. Cochran WG. Sampling Techniques, $3^{\text {rd }}$ Edition. New York: John Wiley \& Sons; 1977.

6. Kneipp SM, Yarandi HN. Complex sampling designs and statistical issues in secondary analysis. West J Nurs Res 2002; 24(5):552-66.

7. Korn EL, Graubard BI. Epidemiologic studies utilizing surveys: accounting for the sampling design. Am J Public Health 1991; 81(9):1166-73.

8. Silva PLN, Pessoa DGCP, Lila MF. Análise estatística de dados da PNAD: incorporando a estrutura do plano amostral. Ciência \& Saúde Coletiva 2002; 7(4): 659-670.

9. Wu C. Optimal calibration estimators in survey sampling. Biometric 2003; 90(4):937-951.

10. Sousa MH, Silva NN. Estimativas obtidas de um levantamento complexo. Rev Saúde Pública 2003; 37(5):662-70.
11. Pessoa DGC, Silva PLN. Análise de dados amostrais complexos. Associação Brasileira de Estatística, São Paulo, 1998: 1-70.

12. Szwarcwald CL, Viacava F. World health survey in Brazil, 2003. Cad Saúde Pública 2005; 21(1 Suppl):S4-5.

13. Lundström S, Särndal CE. Calibration as a Standard Method for Treatment of Nonresponse. Journal of Official Statistics 1999; 15(2):305-327.

14. Kish L. Survey Sampling. Nova Iorque, Wiley, 1965.

15. IBGE - Acesso e utilização de serviços de saúde, 2003. Rio de Janeiro, IBGE, 2005.

16. Vasconcellos MT, Silva PL, Szwarcwald CL. Sampling design for the World Health Survey in Brazil. Cad Saúde Pública 2005; 21(1 Suppl):S89-99.

17. Madow WG. On the theory of systematic sampling, II. Annals of Mathematical Statistics 1949; 20:333-354.

18. Haldane JBS. On a method of estimating frequencies. Biometrika 1945; 33:222-225.

19. Shah BV, Barnwell BG \& Bieler GS. SUDAAN User's Manual. Release 7.5. Research Triangle Park, NC, USA: Research Triangle Institute 1997.

20. Viacava F, Souza-Junior PR, Szwarcwald CL. Coverage of the Brazilian population 18 years and older by private health plans: an analysis of data from the World Health Survey. Cad Saúde Pública 2005; 21(1 Suppl):S119-28.

21. IBGE. Metodologia do Censo Demográfico 2000. Série relatórios metodológicos, 25. Rio de Janeiro: Instituto Brasileiro de Geografia e Estatística; 2003. 